\title{
Nearly optimal time-independent reversal of a spin chain
}

\author{
Aniruddha Bapat $\odot,{ }^{1,2,3,{ }^{*}}$ Eddie Schoute $\odot,{ }^{1,4,5, \dagger}$ Alexey V. Gorshkov $\odot,{ }^{1,2, \ddagger}$ and Andrew M. Childs $\circledast^{1,4,5, \S}$ \\ ${ }^{1}$ Joint Center for Quantum Information and Computer Science, NIST/University of Maryland, College Park, Maryland 20742, USA \\ ${ }^{2}$ Joint Quantum Institute, NIST/University of Maryland, College Park, Maryland 20742, USA \\ ${ }^{3}$ Lawrence Berkeley National Laboratory, Berkeley, California 94720, USA \\ ${ }^{4}$ Department of Computer Science, University of Maryland, College Park, Maryland 20742, USA \\ ${ }^{5}$ Institute for Advanced Computer Studies, University of Maryland, College Park, Maryland 20742, USA
}

(Received 5 April 2020; accepted 20 September 2021; published 22 February 2022)

\begin{abstract}
We propose a time-independent Hamiltonian protocol for the reversal of qubit ordering in a chain of $N$ spins. Our protocol has an easily implementable nearest-neighbor, transverse-field Ising model Hamiltonian with timeindependent, nonuniform couplings. Under appropriate normalization, we implement this state reversal three times faster than a naive approach using SWAP gates, in time comparable to a protocol of Raussendorf [Phys. Rev. A 72, 052301 (2005)] that requires dynamical control. We also prove lower bounds on state reversal by using results on the entanglement capacity of Hamiltonians and show that we are within a factor $1.502(1+1 / N)$ of the shortest time possible. Our lower bound holds for all nearest-neighbor qubit protocols with arbitrary finite ancilla spaces and local operations and classical communication. We give numerical evidence that the fast reversal protocols are more robust to noise than a SWAP-based reversal. Finally, we extend our protocol to an infinite family of nearest-neighbor, time-independent Hamiltonian protocols for state reversal. This includes chains with nearly uniform coupling that may be especially feasible for experimental implementation.
\end{abstract}

DOI: 10.1103/PhysRevResearch.4.L012023

\section{INTRODUCTION}

Quantum information transfer is a fundamental operation in quantum physics, and fast, accurate protocols for transferring quantum states across a physical system are likely to play a key role in the design of quantum computers [1,2]. For example, quantum information transfer can be used to establish long-range entanglement and is also useful for qubit routing in quantum architectures with limited connectivity $[3,4]$. Extensive work has studied the implementation of various information transfer protocols, often via Hamiltonian dynamics on spin chains [5].

Information transfer in Hamiltonian systems is governed by the spread of entanglement and has close links to Lieb-Robinson bounds [6], entanglement area laws [7], and algorithms for quantum simulation [8]. Fundamental limits to the rate of entanglement growth are set by bounds on the asymptotic entanglement capacity [9-12] and more recent small incremental entangling theorems [13-16]. We show that these limits can also be used to obtain lower bounds on the execution time of Hamiltonian protocols for information

\footnotetext{
*ani@1bl.gov

†eschoute@umd.edu

¥gorshkov@umd.edu

§amchilds@umd.edu
}

Published by the American Physical Society under the terms of the Creative Commons Attribution 4.0 International license. Further distribution of this work must maintain attribution to the author(s) and the published article's title, journal citation, and DOI. transfer. This raises the question of whether a protocol can achieve optimality by saturating the bound.

Quantum state transfer studies protocols for moving qubits through a spin chain [17]. Long-range interactions can be used to speed up protocols [18], but here we consider only nearest-neighbor interactions. State transfer protocols usually assume the intermediate medium to be in a known initial state [19-22] or allow it to change in an unknown or nontrivial manner $[23,24]$. Such protocols are not directly applicable when some or all spins in the chain contain data qubits that need to be transferred or maintained.

Protocols for state reversal, also known as state mirroring [25], take steps towards addressing this issue. State reversal reverses any input state on a spin chain about the center of the chain. Specifically, with qubit labeling $1,2, \ldots, N$, state reversal corresponds to the unitary

$$
R:=\prod_{k=1}^{\lfloor N / 2\rfloor} \operatorname{SWAP}_{k, N+1-k}
$$

up to a global phase, which is independent of the state. State reversal is potentially a useful subroutine for the more general task of qubit routing, where we wish to apply arbitrary permutations to the qubits. Early results in this area require the state to be in the single-excitation subspace [26] or introduce phases in the final state that depend on a nonlocal property such as the number of qubits in state $|1\rangle[25,27]$. The protocol introduces a phase $(-1)^{M(M-1) / 2}$ that is a function of the excitation number $M(\bmod 4)$. This is nontrivial to correct: Consider the task of signaling the value of the left bit to the right end of a chain with zeros in the bulk. A right edge state prepared in |\rangle$+$ is flipped to $|-\rangle$ by the phase correction 
procedure, conditioned on the value of the left bit. By the signaling lower bound, we incur a time overhead linear in $N$ to correct these phases and implement a reversal for a general state. These limitations were later removed by time-dependent protocols for state reversal [28-30]. Concepts from [28,29] can also be used for translation-invariant universal quantum computation by, for example, modeling a quantum cellular automaton [28,31-33].

In this work, we propose a time-independent protocol for state reversal using nearest-neighbor interactions that we expect has applications in noisy, connectivity-limited quantum devices. We show that the execution time of our protocol is nearly optimal, comparable to the time-dependent protocol given in [28]. We also find, through simulations, that these reversal protocols have reduced error scaling in system size to noise due to static disorder caused by imperfect fabrication when compared to a SWAP-based protocol (see Supplemental Material [34]). In addition, our protocol does not require dynamical control but only engineered nearest-neighbor couplings, so we expect it to be more experimentally feasible on near-term quantum systems such as superconducting qubits [35] where dynamical control could be an additional source of noise.

Before presenting our state reversal protocol in more detail, let us elaborate on the claim that it is nearly optimalspecifically, that it has an evolution time within a factor $1.502(1+1 / N)$ of the shortest possible. For any nearestneighbor spin Hamiltonian $H$, a timescale follows from a normalization that limits the strength of every two-qubit interaction but allows fast local operations. Up to local unitaries, we can write any two-qubit Hamiltonian in the canonical form [36]

$$
K:=\sum_{j \in\{x, y, z\}} \mu_{j} \sigma_{j} \otimes \sigma_{j},
$$

where $\mu_{x} \geqslant \mu_{y} \geqslant\left|\mu_{z}\right| \geqslant 0$ and $\sigma_{j}$ are the Pauli matrices. We impose the normalization condition that $\|K\|=\sum_{j}\left|\mu_{j}\right| \leqslant 1$ for all interactions, where $\|\cdot\|$ is the spectral norm. Under this normalization, a SWAP can be optimally implemented in time $3 \pi / 4$ [37], and our protocol achieves state reversal in time

$$
t_{N}:=\pi \sqrt{(N+1)^{2}-p(N)} / 4,
$$

where $p(N):=N(\bmod 2)$. This is equivalent in time to a SWAP gate circuit of depth $\sim N / 3$. As state reversal using only SWAPs requires depth at least $N-1$ [38], our protocol is faster than any SWAP-based protocol by an asymptotic factor of 3 . Similarly, we can compare to other time-independent Hamiltonian protocols that use nearest-neighbor interactions: [19] implements state transfer in time $N \pi / 4$ and [25] implements state reversal in time $N \pi / 2$ but introduces relative phases in the state as mentioned earlier. Our time-independent protocol (and some time-dependent protocols [28-30]) thus improve upon these previous protocols for state transfer and state reversal except for a subleading term.

We lower-bound the time for state reversal, which can generate entanglement across a bipartition, by using bounds on the asymptotic entanglement capacity in a more general model $[10,12]$. The asymptotic entanglement capacity bounds the rate at which entanglement can be generated by any evolution

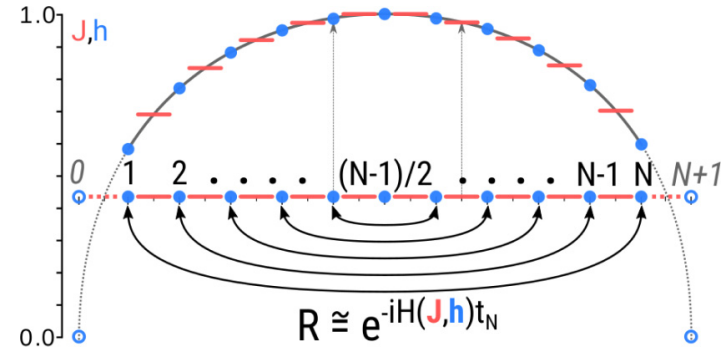

FIG. 1. The state reversal operation $R$ (depicted by arrows) and an illustration of our time-independent protocol to implement it. The nearest-neighbor $\sigma_{x}^{k} \sigma_{x}^{k+1}$ couplings $\left(J_{k}\right.$, dashes) and on-site $\sigma_{z}^{k}$ fields $\left(h_{k}\right.$, dots) are plotted on the $y$ axis. Sites $0, N+1$ are ancilla qubits, which are not part of the protocol and are used purely in the analysis.

of a given bipartite Hamiltonian interspersed with arbitrary local operations and classical communication (LOCC) and with arbitrary finite local ancilla spaces. We give an explicit example of entanglement generated by state reversal and lower-bound the time using the capacity of a normalized two-qubit interaction in canonical form (2), even allowing for LOCC. Nonetheless, our state reversal protocol is able to nearly saturate this bound without classical communication, without ancillas, and with only nearest-neighbor interactions throughout the chain.

We propose a state reversal protocol with Hamiltonian of the form

$$
H(\boldsymbol{J}, \boldsymbol{h})=J_{0} \sigma_{x}^{1}+\sum_{k=1}^{N-1} J_{k} \sigma_{x}^{k} \sigma_{x}^{k+1}+J_{N} \sigma_{x}^{N}-\sum_{k=1}^{N} h_{k} \sigma_{z}^{k},
$$

where the coefficients $\boldsymbol{J}, \boldsymbol{h}$ are engineered as follows. Letting

$$
a_{k}:=\pi \sqrt{(N+1)^{2}-(N+1-k)^{2}} /\left(4 t_{N}\right),
$$

for $k \in \mathbb{N}$, our protocol is defined as follows (see also Fig. 1).

Protocol 1. Let $J_{k}=a_{2 k+1}, h_{k}=a_{2 k}$ for all sites $k$, and let $H:=H(\boldsymbol{J}, \boldsymbol{h})$. Apply $U:=e^{-i t_{N} H}$ to the input state.

We show in the following sections that our protocol implements state reversal exactly, up to a global phase (we denote this equivalence by $\cong$ ).

Theorem 1. $U \cong R$.

\section{A. Proof and analysis of the protocol}

We prove the correctness of our protocol (i.e., Theorem 1) by mapping the spin chain to a doubled chain of Majorana fermions via a Jordan-Wigner transformation, describing the action in the Majorana picture, and then mapping back to the spin picture. To help with the analysis, we extend the chain with two ancillary sites $\{0, N+1\}$ called the edge, $E$, and refer to the sites $\{1, \ldots, N\}$ as the bulk, $B$. We define the transverse-field Ising model Hamiltonian

$$
\widetilde{H}:=\sum_{k=0}^{N} a_{2 k+1} \sigma_{x}^{k} \sigma_{x}^{k+1}-\sum_{k=1}^{N} a_{2 k} \sigma_{z}^{k} .
$$

on the extended chain that reduces to $H$ when the edge is initialized to state $|++\rangle$. Similarly, we define $\widetilde{U}:=e^{-i \widetilde{H_{N}}}$. Note that the operator $\widetilde{H}$ (and hence $\widetilde{U}$ ) acts trivially on $|++\rangle_{E}$, so this edge state does not change through the course of the 
evolution. [Our results also hold using the edge state $|--\rangle_{E}$, which is equivalent to negating the sign of the longitudinal fields in (4).] We then prove that in the Heisenberg picture, Pauli matrices on site $k$ map to the corresponding Pauli on site $N+1-k$ for all sites $k$ in the chain.

First, we map to the doubled chain of Majorana fermionic operators by defining

$$
\gamma_{2 k}:=P_{[0, k-1]} \sigma_{x}^{k}, \quad \gamma_{2 k+1}:=P_{[0, k-1]} \sigma_{y}^{k}
$$

at each site, where we have used the notation $P_{[a, b]}:=$ $\prod_{j=a}^{b}\left(-\sigma_{z}^{j}\right)$ for the Jordan-Wigner parity string between sites $a$ and $b$. The $\gamma_{k}$ are Hermitian and satisfy the Majorana anticommutation relations $\left\{\gamma_{j}, \gamma_{k}\right\}=2 \delta_{j k}$. We also see that $\sigma_{z}^{k}=-i \gamma_{2 k} \gamma_{2 k+1}$ and $\sigma_{x}^{k} \sigma_{x}^{k+1}=i \gamma_{2 k+1} \gamma_{2 k+2}$, leading (6) to take the form

$$
\widetilde{H}=i \sum_{k=1}^{2 N+1} a_{k} \gamma_{k} \gamma_{k+1}
$$

The Majoranas $\gamma_{0}, \gamma_{2 N+3}$ do not appear in the sum, since $a_{0}=a_{2 N+2}=0$. In the following lemma, we show how $\widetilde{U}$ transforms the Majorana operators. Our main technique is an analogy with the dynamics of the $y$ component of the spin operator for a spin $N+\frac{1}{2}$ particle, similar to [19,25]. Here, the same analogy provides a protocol which gives state reversal on all spins in the chain without introducing relative phases.

Lemma 1. The operation $\widetilde{U}$ acts on the Majorana operators as

$$
\widetilde{U} \gamma_{k} \widetilde{U}^{\dagger}= \begin{cases}\gamma_{k} & \text { if } k=0,2 N+3 \\ (-1)^{k-1} \gamma_{2 N+3-k} & \text { otherwise. }\end{cases}
$$

Proof. For the first case, $\widetilde{H}$ has no overlap with operators $\gamma_{0}$ and $\gamma_{2 N+3}$, so they are stationary under evolution by $\widetilde{H}$.

For the remaining cases, we use the analogy with a spin $s=N+\frac{1}{2}$ particle. The Heisenberg evolution of $\gamma_{k}$ corresponds to the rotation of the $S_{z}$ eigenstate $|s, k-s-1\rangle$ of magnetization $k-s-1$. Observing that

$$
\frac{i \pi}{4 t_{N}}\left\langle s, m\left|S_{y}\right| s, m^{\prime}\right\rangle=a_{s+m+1}\left(\delta_{m^{\prime}(m+1)}-\delta_{m\left(m^{\prime}+1\right)}\right)
$$

(with $\hbar=1$ ), we can express (8) in the bilinear form $\widetilde{H}=$ $\frac{1}{2} \boldsymbol{\gamma}^{\dagger} A \boldsymbol{\gamma}$, for the vector $\boldsymbol{\gamma}:=\left[\begin{array}{llll}\gamma_{1} & \gamma_{2} & \ldots & \gamma_{2 N+2}\end{array}\right]$ and the matrix $A:=-\pi /\left(2 t_{N}\right) S_{y}$ expressed in the $S_{z}$ basis. Using the Majorana commutation relations, we have $\dot{\gamma}=i[\widetilde{H}, \gamma]=$ $2 i A \boldsymbol{\gamma}$, so $\boldsymbol{\gamma}(t)=e^{2 i A t} \boldsymbol{\gamma}(0)$. The Heisenberg evolution of $\gamma_{k}$ under $\widetilde{H}$ for time $t_{N}$ is exactly analogous to the (Schrödinger) time evolution of the state $|s, k-s-1\rangle$ under $S_{y}$ for time $\pi$. A $\pi$ rotation under $S_{y}$ maps

$$
|s,-s+k-1\rangle \mapsto(-1)^{k-1}|s, s-k+1\rangle,
$$

and correspondingly, $\gamma_{k}\left(t_{N}\right)=(-1)^{k-1} \gamma_{2 N+3-k}$.

Note that eq. 11 can easily be verified for a spin-1/2 particle. Similarly, a spin- $s$ particle may be viewed as a system of $2 s$ spin- $\frac{1}{2}$ particles with maximal total spin. In this picture, a $\pi$ rotation under $S_{y}$ corresponds to independent $\pi$ rotations of each small spin. Since the state $|s, k-s-1\rangle$ is represented by a permutation-symmetric state with $k-1$ up spins, the $\pi$ rotation maps it to a state with $2 s-(k-1)$ up spins and introduces a phase $(-1)$ for each up spin, which is precisely (11).
Due to the signed reversal of the Majoranas in Lemma 1, the parity string $P_{[0, k]}=i^{b+1-a} \prod_{j=2 a}^{2 b+1} \gamma_{j}$ is (with the exception of $\gamma_{0}$ ) reflected about the center of the chain with an overall phase that exactly cancels when the product is reordered by increasing site index. The invariance of the edge Majoranas is crucial, as it provides a phase factor that cancels the state-dependent phases when we revert to the spin picture. In particular, we have the following lemma.

Lemma 2. The operation $\widetilde{U}$ acts on the parity strings as $\widetilde{U} P_{[0, k]} \widetilde{U}^{\dagger}=i \sigma_{x}^{0} \sigma_{x}^{N+1} P_{[0, N-k]}$ for all $k$.

Proof. Applying Lemma 1, we have

$$
\begin{aligned}
\widetilde{U} P_{[0, k]} \widetilde{U}^{\dagger} & =i^{k+1}(-1)^{k(2 k+1)} \gamma_{0} \prod_{j=1}^{2 k+1} \gamma_{2 N+3-j} \\
& =\gamma_{0} P_{[0, N]} P_{[0, N-k]} \gamma_{2 N+2},
\end{aligned}
$$

where we reordered the product and used $P_{[N+1-k, N]}=$ $P_{[0, N]} P_{[0, N-k]}$. From the Majorana anticommutation relations and (7), the result follows.

Now we prove the main theorem.

Proof of Theorem 1. $U \cong R$ holds iff all bulk observables on the chain transform identically under $U, R$. For any operator $\mathcal{O}^{k}$ supported on bulk site $k \in\{1, \ldots, N\}$, we show that $U \mathcal{O}^{k} U^{\dagger}=\left\langle++\left|\widetilde{U} \mathcal{O}^{k} \widetilde{U}^{\dagger}\right|++\right\rangle_{E}=\mathcal{O}^{N+1-k}$. (Henceforth we drop the edge subscript $E$.) By Lemmas 1 and $2, \sigma_{x}^{k}$ is mapped to

$$
\begin{gathered}
U \sigma_{x}^{k} U^{\dagger}=\left\langle++\left|\widetilde{U} P_{[0, k-1]} \gamma_{2 k} \tilde{U}^{\dagger}\right|++\right\rangle \\
=-i\left\langle++\left|\sigma_{x}^{0} \sigma_{x}^{N+1} P_{[0, N+1-k]} \gamma_{2 N+3-2 k}\right|++\right\rangle \\
=-i \sigma_{z}^{N+1-k} \sigma_{y}^{N+1-k}=\sigma_{x}^{N+1-k} .
\end{gathered}
$$

Next, we use Lemma 2 to show that $\sigma_{z}^{k}$ is mapped to

$$
\begin{gathered}
U \sigma_{z}^{k} U^{\dagger}=-\left\langle++\left|\widetilde{U} P_{[0, k-1]} P_{[0, k]} \widetilde{U}^{\dagger}\right|++\right\rangle \\
=\left\langle++\left|\sigma_{x}^{0} \sigma_{x}^{N+1} P_{[0, N+1-k]} \sigma_{x}^{0} \sigma_{x}^{N+1} P_{[0, N-k]}\right|++\right\rangle \\
=\sigma_{z}^{N+1-k} .
\end{gathered}
$$

All other observables can be written in terms of the on-site Pauli operators $\sigma_{x}^{k}, \sigma_{z}^{k}$, so $U$ is identical to $R$, up to global phase.

\section{B. Time lower bound}

We now prove a lower bound on the optimal time, $t^{*}$, to implement state reversal using normalized local interactions. Let the entanglement entropy between systems $A$ and $B$ of a bipartite state $|\psi\rangle_{A B}$ be $E(|\psi\rangle)$, defined as the local von Neumann entropy $S(\rho):=-\operatorname{Tr} \rho \log _{2} \rho$, for $\rho=\operatorname{Tr}_{B}|\psi\rangle\langle\psi|$. Then, the asymptotic entanglement capacity of a Hamiltonian $H$ that couples systems $A$ and $B$ was shown to equal [12]

$$
E_{H}=\sup _{|\psi\rangle \in \mathcal{H}_{A A^{\prime} B B^{\prime}}} \lim _{t \rightarrow 0} \frac{\mathrm{E}\left(e^{-i H t}|\psi\rangle\right)-\mathrm{E}(|\psi\rangle)}{t},
$$

where $\mathcal{H}_{A A^{\prime} B B^{\prime}}$ is the Hilbert space of the bipartite systems $A$ and $B$ with arbitrarily large ancilla spaces $A^{\prime}$ and $B^{\prime}$, respectively. In particular, for a Hamiltonian of the form $\sigma_{x} \otimes \sigma_{x}$, 
$[9,10]$ showed that

$$
\alpha:=E_{\sigma_{x} \otimes \sigma_{x}}=2 \max _{y} \sqrt{y(1-y)} \log _{2} \frac{y}{1-y} \approx 1.912 .
$$

This is tighter than the more general small incremental entangling bound $E_{H} \leqslant c\|H\| \log _{2} d=2$ for the conjectured $c=2$ [13] (best known $c=4$ [15]) and where the smallest dimension of $A$ or $B$ gives $d=2$. Since $E$ is invariant under local unitaries, a direct corollary is that $E_{\sigma_{y} \otimes \sigma_{y}}=E_{\sigma_{z} \otimes \sigma_{z}}=\alpha$.

We now show that Protocol 1 is close to the shortest time possible.

Theorem 2. It holds that $\frac{t_{N}}{t^{*}(1+1 / N)} \leqslant \alpha \pi / 4<1.502$.

Proof. We prove the time lower bound via an upper bound on the rate of increase of entanglement across a cut in the center of the chain (allowing differences of one qubit for odd $N)$. Designate the left half of the cut as subsystem $\mathcal{A}$ and the right half as subsystem $\mathcal{B}$. $\mathcal{A}$ consists of subsystem $A$ given by the qubit at site $\lfloor N / 2\rfloor$ adjacent to the cut, and subsystem $A^{\prime}$ consisting of the remaining qubits to the left of the cut as well as a finite but arbitrary number of ancilla systems that are not part of the chain. Similarly, $\mathcal{B}$ consists of subsystem $B$, the qubit at site $\lfloor N / 2\rfloor+1$, and $B^{\prime}$, the remaining qubits in the right half with an arbitrary finite number of ancilla.

Consider Hamiltonians of the form $H(t)=K(t)+\bar{K}(t)$ specifying the evolution of the $\mathcal{A B}$ system, where $K(t)$ is a two-qubit Hamiltonian supported on systems $A B$ (i.e., the cut edge), while $\bar{K}$ contains terms supported on $A A^{\prime}$ or $B B^{\prime}$ but not the cut edge $A B$. For brevity, we drop the time parameter $t$ even though we allow the Hamiltonian to be time dependent. We assume that $K$ is expressed in canonical form (2) due to equivalence under local unitaries. Aside from its support, we make no assumptions about the form of $\bar{K}$ (so the resulting bound is more general than nearest-neighbor interactions). We call $H$ satisfying these conditions divisible and also call protocols using divisible Hamiltonians divisible.

Observing that $E_{H}$ is the supremum over a time derivative of the von Neumann entropy of $\rho=\operatorname{Tr}_{\mathcal{B}}|\psi\rangle\langle\psi|$, we have

$$
\begin{gathered}
E_{H}=\sup _{|\psi\rangle} \operatorname{Tr}\left(-\frac{d \rho}{d t} \log _{2} \rho-\rho \frac{d \log _{2} \rho}{d t}\right) \\
=\sup _{|\psi\rangle} \operatorname{Tr}\left(-\frac{d \rho}{d t} \log _{2} \rho\right) .
\end{gathered}
$$

The reduced density matrix $\rho$ has time evolution

$$
\frac{d \rho}{d t}=-i \operatorname{Tr}_{\mathcal{B}}[H,|\psi\rangle\langle\psi|]
$$

We substitute $H=\bar{K}+\sum_{j \in\{x, y, z\}} \mu_{j} \sigma_{j} \otimes \sigma_{j}$ in the commutator and substitute the time dependence of $\rho$ into eq. 23. By linearity of the trace and sublinearity of the supremum, we get

$$
E_{H} \leqslant E_{\bar{K}}+\sum_{j \in\{x, y, z\}} \mu_{j} E_{\sigma_{j} \otimes \sigma_{j}} \leqslant \alpha,
$$

where we observe that $E_{\bar{K}}=0$ since $\bar{K}$ does not have support across the cut, and use the normalization condition $\sum_{j}\left|\mu_{j}\right| \leqslant$ 1. This bound holds for all divisible Hamiltonians $H$, with nearest-neighbor Hamiltonians as a special case.

The entanglement generated by any divisble protocol can now be bounded in time. We observe that if the protocol contains local measurements, then these cannot increase entanglement $E(|\psi\rangle)$ and that feedback may be viewed as a particular time dependence of $H$ conditioned on measurement outcomes. Therefore, (25) bounds the total increase in entanglement across bipartition $\mathcal{A B}$ over a time $t^{*}$ by

$$
E\left(\left|\psi\left(t^{*}\right)\right\rangle\right)-E(|\psi(0)\rangle) \leqslant \alpha t^{*}
$$

for any initial state $|\psi(0)\rangle$ acted on by a divisible protocol and LOCC.

Finally, we give an explicit bound on the worst-case time of divisible state reversal protocols by specifying an initial state. Let the system start in the product state $|\phi\rangle_{\mathcal{A}} \otimes|\phi\rangle_{\mathcal{B}}$ where each qubit forms a Bell state with a local ancilla not part of the chain. Clearly, $E\left(|\phi\rangle_{\mathcal{A}}\right) \otimes|\phi\rangle_{\mathcal{B}}=0$. We perform a reversal $R$ on the chain and get the state $|\psi\rangle_{\mathcal{A B}}:=R\left(|\phi\rangle_{\mathcal{A}} \otimes|\phi\rangle_{\mathcal{B}}\right)$, which is maximally entangled, i.e., $E\left(|\psi\rangle_{\mathcal{A B}}\right)=N$. Then, (26) gives the bound

$$
t^{*} \geqslant \frac{E\left(|\psi\rangle_{\mathcal{A B}}\right)-E\left(|\phi\rangle_{\mathcal{A}} \otimes|\phi\rangle_{\mathcal{B}}\right)}{\alpha} \geqslant \frac{N}{\alpha}
$$

on any divisible state reversal protocol. Comparing this to our protocol time (3), we have

$$
\frac{t_{N}}{t^{*}} \leqslant \frac{\alpha \pi \sqrt{(N+1)^{2}-p(N)}}{4 N} \leqslant \frac{\alpha \pi(1+1 / N)}{4} .
$$

\section{Discussion}

The time-dependent protocol in [28] is closely related to our time-independent protocol, and both can be described within the same framework (see Supplemental Material [34]). In the time-dependent case, the state is evolved alternately under two restrictions of the Hamiltonian (4): $H(\mathbf{1}, \mathbf{0})$ (uniform Ising) and $H(\mathbf{0}, \mathbf{1})$ (uniform transverse field), each for time $\pi / 4$, for a total of $N+1$ rounds. In the Majorana picture, these Hamiltonians carry out a simultaneous braiding of neighboring Majoranas along even (respectively, odd) edges of the doubled Majorana chain. The resulting map matches Lemma 1 exactly, implying that the two protocols are identical at the level of Majorana operators. Indeed, any protocol achieving the map in Lemma 1 is guaranteed to implement state reversal.

In fact, as shown in the Supplemental Material [34], there is an infinite family of nearest-neighbor, timeindependent Hamiltonian protocols for state reversal that generalizes Protocol 1. This family is parametrized by a non-negative integer $m$, with modified $\sigma_{x}^{k} \sigma_{x}^{k+1}$ coupling $J_{k}^{(m)} \propto \sqrt{(2 N+1-2 k+4 m)(2 k+1+4 m)}$ and unmodified $\sigma_{z}^{k}$ field strength. Protocol 1 corresponds to the special case of $m=0$. By choosing large $m$, the coupling strength can be engineered to be nearly uniform throughout the chain, which may be a desirable feature in experimental implementations of the protocol [27].

Moreover, we show by numerical simulations that our protocol is more robust to noise. Specifically, we consider static disorder, which could be caused by imperfect fabrication or tuning in noisy, intermediate-scale quantum device implementations (see Supplemental Material [34]). Our results imply, for example, that with strong disorder and an error threshold of 0.03 , a SWAP protocol can only reverse four sites, whereas the time-independent protocol can reverse eight sites. 
In general, we would like to know how fast we can perform qubit routing on graphs. Qubit routing is a key subroutine in quantum architectures with incomplete connectivity [4], and can potentially improve runtimes of general quantum algorithms by overcoming limitations imposed by the underlying qubit connectivity. Indeed, we later showed [39] that a constant-factor speedup over a SWAP-based approach is achievable for general qubit routing on the chain using our fast reversal protocol as a primitive. While a superconstant speedup is not possible in one dimension, our techniques suggest that routing protocols for higher-dimensional systems might be found by exploiting similar mappings between spins and fermions [40-42]. While state transfer in these systems has been studied [43], the more general questions of upper and lower bounds on routing remain open, and our bounds based on the entanglement capacity might yield new insights.

\section{ACKNOWLEDGMENTS}

A.B. and A.G. acknowledge support by the DoE ASCR Quantum Testbed Pathfinder program (Award No. DESC0019040), DOE ASCR Accelerated Research in Quantum Computing Program (Award No. DE-SC0020312), US Department of Energy Award No. DE-SC0019449, NSF PFCQC program, AFOSR, ARO MURI, ARL CDQI, and NSF PFC at JQI. E.S. and A.C. acknowledge support by the DOE ASCR Quantum Testbed Pathfinder program (Award No. DE-SC0019040) and the ARL (MURI Award No. W911NF16-1-0349).
[1] D. P. DiVincenzo, The physical implementation of quantum computation, Fortschr. Phys. 48, 771 (2000).

[2] H. J. Kimble, The quantum internet, Nature (London) 453, 1023 (2008).

[3] R. Beals, S. Brierley, O. Gray, A. W. Harrow, S. Kutin, N. Linden, D. Shepherd, and M. Stather, Efficient distributed quantum computing, Proc. R. Soc. London, Ser. A 469, 20120686 (2013).

[4] A. M. Childs, E. Schoute, and C. M. Unsal, Circuit transformations for quantum architectures, in 14th Conference on the Theory of Quantum Computation, Communication and Cryptography (TQC 2019), Leibniz International Proceedings in Informatics (LIPIcs), Vol. 135, edited by W. van Dam and L. Mancinska (Schloss Dagstuhl-Leibniz-Zentrum fuer Informatik, Dagstuhl, Germany, 2019), pp. 3:1-3:24.

[5] S. Bose, Quantum communication through spin chain dynamics: An introductory overview, Contemp. Phys. 48, 13 (2007).

[6] E. H. Lieb and D. W. Robinson, The finite group velocity of quantum spin systems, Statistical Mechanics (Springer, New York, 1972), pp. 425-431.

[7] J. Eisert, M. Cramer, and M. B. Plenio, "Colloquium: Area laws for the entanglement entropy,” Rev. Mod. Phys. 82, 277 (2010).

[8] J. Haah, M. Hastings, R. Kothari, and G. H. Low, Quantum algorithm for simulating real time evolution of lattice Hamiltonians, in 2018 IEEE 59th Annual Symposium on Foundations of Computer Science (FOCS) (IEEE, New York, 2018), pp. 350-360.

[9] W. Dür, G. Vidal, J. I. Cirac, N. Linden, and S. Popescu, Entanglement Capabilities of Nonlocal Hamiltonians, Phys. Rev. Lett. 87, 137901 (2001).

[10] A. M. Childs, D. W. Leung, F. Verstraete, and G. Vidal, Asymptotic entanglement capacity of the Ising and anisotropic Heisenberg interactions, Quantum Inf. Comput. 3, 97 (2003).

[11] A. M. Childs, D. W. Leung, and G. Vidal, Reversible simulation of bipartite product Hamiltonians, IEEE Trans. Inf. Theory 50, 1189 (2004).

[12] C. H. Bennett, A. W. Harrow, D. W. Leung, and J. A. Smolin, On the capacities of bipartite Hamiltonians and unitary gates, IEEE Trans. Inf. Theory 49, 1895 (2003).

[13] S. Bravyi, Upper bounds on entangling rates of bipartite Hamiltonians, Phys. Rev. A 76, 052319 (2007).
[14] K. Van Acoleyen, M. Mariën, and F. Verstraete, Entanglement Rates and Area Laws, Phys. Rev. Lett. 111, 170501 (2013).

[15] K. M. R. Audenaert, Quantum skew divergence, J. Math. Phys. 55, 112202 (2014).

[16] M. Mariën, K. M. R. Audenaert, K. Van Acoleyen, and F. Verstraete, Entanglement rates and the stability of the area law for the entanglement entropy, Commun. Math. Phys. 346, 35 (2016).

[17] S. Bose, Quantum Communication through an Unmodulated Spin Chain, Phys. Rev. Lett. 91, 207901 (2003).

[18] G. Gualdi, V. Kostak, I. Marzoli, and P. Tombesi, Perfect state transfer in long-range interacting spin chains, Phys. Rev. A 78, 022325 (2008).

[19] M. Christandl, N. Datta, A. Ekert, and A. J. Landahl, Perfect State Transfer in Quantum Spin Networks, Phys. Rev. Lett. 92, 187902 (2004).

[20] M. Christandl, N. Datta, T. C. Dorlas, A. Ekert, A. Kay, and A. J. Landahl, Perfect transfer of arbitrary states in quantum spin networks, Phys. Rev. A 71, 032312 (2005).

[21] L. Campos Venuti, C. Degli Esposti Boschi, and M. Roncaglia, Qubit Teleportation and Transfer Across Antiferromagnetic Spin Chains, Phys. Rev. Lett. 99, 060401 (2007).

[22] L. Banchi, A. Bayat, P. Verrucchi, and S. Bose, Nonperturbative Entangling Gates between Distant Qubits Using Uniform Cold Atom Chains, Phys. Rev. Lett. 106, 140501 (2011).

[23] N. Y. Yao, L. Jiang, A. V. Gorshkov, Z.-X. Gong, A. Zhai, L.-M. Duan, and M. D. Lukin, Robust Quantum State Transfer in Random Unpolarized Spin Chains, Phys. Rev. Lett. 106, 040505 (2011).

[24] C. Di Franco, M. Paternostro, and M. S. Kim, Perfect State Transfer on a Spin Chain without State Initialization, Phys. Rev. Lett. 101, 230502 (2008).

[25] C. Albanese, M. Christandl, N. Datta, and A. Ekert, Mirror Inversion of Quantum States in Linear Registers, Phys. Rev. Lett. 93, 230502 (2004).

[26] T. Shi, Y. Li, Z. Song, and C.-P. Sun, Quantum-state transfer via the ferromagnetic chain in a spatially modulated field, Phys. Rev. A 71, 032309 (2005).

[27] P. Karbach and J. Stolze, Spin chains as perfect quantum state mirrors, Phys. Rev. A 72, 030301(R) (2005). 
[28] R. Raussendorf, Quantum computation via translation-invariant operations on a chain of qubits, Phys. Rev. A 72, 052301 (2005).

[29] J. Fitzsimons and J. Twamley, Globally Controlled Quantum Wires for Perfect Qubit Transport, Mirroring, and Computing, Phys. Rev. Lett. 97, 090502 (2006).

[30] P. Kumar and S. Daraeizadeh, Parity-based mirror inversion for efficient quantum state transfer and computation in nearestneighbor arrays, Phys. Rev. A 91, 042310 (2015).

[31] D. T. Stephen, H. P. Nautrup, J. Bermejo-Vega, J. Eisert, and R. Raussendorf, Subsystem symmetries, quantum cellular automata, and computational phases of quantum matter, Quantum 3, 142 (2019).

[32] T. Devakul and D. J. Williamson, Universal quantum computation using fractal symmetry-protected cluster phases, Phys. Rev. A 98, 022332 (2018).

[33] R. Raussendorf, C. Okay, D.-S. Wang, D. T. Stephen, and H. P. Nautrup, Computationally Universal Phase of Quantum Matter, Phys. Rev. Lett. 122, 090501 (2019).

[34] See Supplemental Material at http://link.aps.org/supplemental/ 10.1103/PhysRevResearch.4.L012023 for a generalization of Protocol 1, connections to the time-dependent protocol of [28], and an analysis of the performance of reversal protocols in the presence of disorder.

[35] M. Kjaergaard, M. E. Schwartz, J. Braumüller, P. Krantz, J. I.J. Wang, S. Gustavsson, and W. D. Oliver, Superconducting qubits: Current state of play, Annu. Rev. Condens. Matter Phys. 11, 369 (2020).

[36] C. H. Bennett, J. I. Cirac, M. S. Leifer, D. W. Leung, N. Linden, S. Popescu, and G. Vidal, Optimal simulation of two-qubit Hamiltonians using general local operations, Phys. Rev. A 66, 012305 (2002).

[37] G. Vidal, K. Hammerer, and J. I. Cirac, Interaction Cost of Nonlocal Gates, Phys. Rev. Lett. 88, 237902 (2002).

[38] N. Alon, F. R. K. Chung, and R. L. Graham, Routing permutations on graphs via matchings, SIAM J. Discrete Math. 7, 513 (1994).

[39] A. Bapat, A. M. Childs, A. V. Gorshkov, S. King, E. Schoute, and $\mathrm{H}$. Shastri, Quantum routing with fast reversals, Quantum 5, 533 (2021).

[40] H. Yao and S. A. Kivelson, Exact Chiral Spin Liquid with Non-Abelian Anyons, Phys. Rev. Lett. 99, 247203 (2007).

[41] A. Kitaev, Anyons in an exactly solved model and beyond, Ann. Phys. 321, 2 (2006).

[42] Y.-A. Chen, Exact bosonization in arbitrary dimensions, Phys. Rev. Res. 2, 033527 (2020).

[43] N. Y. Yao, C. R. Laumann, A. V. Gorshkov, H. Weimer, L. Jiang, J. I. Cirac, P. Zoller, and M. D. Lukin, Topologically protected quantum state transfer in a chiral spin liquid, Nat. Commun. 4, 1585 (2013). 